\title{
The first reported case of a variant of Mal de Maleda of the Gamborg-Nielsen type in an Egyptian origin patient
}

\author{
Khalid M. Al-Husain', Ahmed A. Al-Thubaiti', Farah M. Alzahrani², Iqbal A. Bukhari', \\ Mohammad El-Shawarby ${ }^{4}$
}

${ }^{1}$ College of Medicine, Imam Abdulrahman Bin Faisal University (IAU), Dammam, Kingdom of Saudi Arabia, ${ }^{2}$ College of Medicine, Arabian Gulf University (AGU), Bahrain, ${ }^{3}$ Dermatology Department, College of Medicine, Imam Abdulrahman Bin Faisal University (IAU) and King Fahd Hospital of the University, Dammam, Kingdom of Saudi Arabia, ${ }^{4}$ Pathology Department, College of Medicine, Imam Abdulrahman Bin Faisal University (IAU) and King Fahd Hospital of the University, Dammam, Kingdom of Saudi Arabia

Corresponding author: Prof. Iqbal A. Bukhari, E-mail: ibukhari@iau.edu.sa

\begin{abstract}
Mal de Meleda is a rare genodermatosis with an autosomal recessive inheritance. Mutations in the SLURP1 gene are the cause of this disease. Clinically, it is characterized by progressive palmoplantar hyperkeratosis exhibiting a transgradiens pattern extending to the dorsal aspects of the hands and feet in a glove and stocking pattern. It is also associated with hyperhidrosis, nail changes, subungual hyperkeratosis and perioral erythema. Here we report the first case of Gamborg-Nielsen variant of Mal de Meleda disorder in a patient of an Egyptian origin.
\end{abstract}

Key words: Mal de Meleda; Keratoderma; Genodermatosis; SLURPl gene

\section{INTRODUCTION}

Mal de Meleda is a type of palmoplantar keratoderma (PPK) which has an autosomal recessive (AR) pattern of inheritance. Clinically, the patient present with bilateral diffuse PPK in a stocking and glove pattern with sharp margins and a yellow tone complicated by hyperhidrosis and microbial infection leading to malodorous and painful lesions [1]. Nail anomalies are the most common associated feature in the form of subungual hyperkeratosis, Beau's lines, onycholysis, hyperconvexity, and koilonychias [1,2]. Other associated features such as psoriasiform lesions on the knees and elbows, perioral erythema, angular cheilitis, arched palate and rarely malignant melanoma within the areas of hyperkeratosis may be seen [3-7]. Mal de Meleda can be diagnosed based on clinical features, family history and genetic analysis. Here we report the first case of Gamborg-Nielsen variant of Mal de Meleda in an Egyptian origin patient in the English medical literature.

\section{CASE REPORT}

A 13-year-old Egyptian boy presented to the dermatology clinic of King Fahd Hospital of the University with erythematous patches affecting the palms and soles. The condition started at the age of 3 months when he developed palmoplantar erythematous erosive lesions with fluctuating course. There was no history of blisters or fissures. That time he was seen by several dermatologists and treated with oral antihistamines and topical steroids. According to the parents the condition worsened and became palmoplantar macerated hyperkeratotic lesions. Past medical history was irrelevant. There was no history of atopy. The mother and father are first degree relatives with no similar condition reported in the family. The

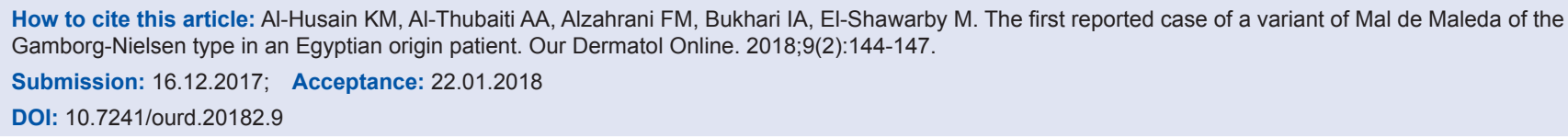


patient had normal growth milestones and doing well in school. On examination there was bilateral symmetrical palmoplantar waxy hyperkeratotic lesions with irregular borders extending to the dorsum of both hands and feet with erythematous background (Figs. 1 and 2). There was hyperhidrosis that was not malodorous. There were no nail changes or contractures or autoamputation of the digits. The face showed scaly slightly erythematous patches on the cheeks and nose. The lips were slightly dry without perioral erythema. His gingiva, teeth and tongue were normal. Hair was normal. Besides, there was erythematous hyperkeratotic patches on the extensor surfaces of the arms, legs, thighs and back but the knees and elbows were free of lesions. There were no hearing loss or eye abnormalities. Systemic examination showed normal findings. Laboratory blood tests including complete blood count, liver function test,

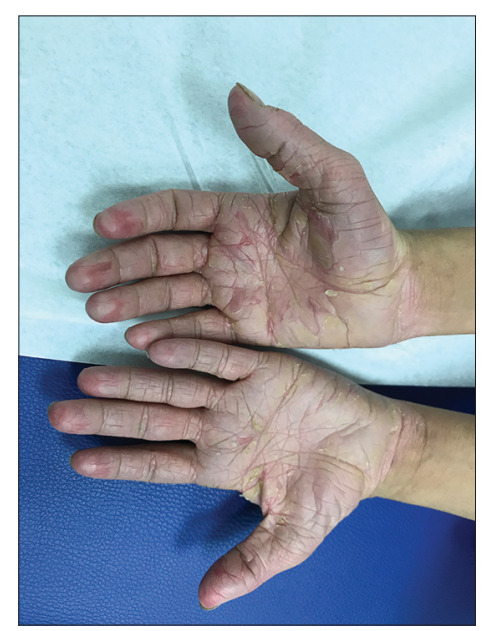

Figure 1: Bilateral thickened palmar keratoderma with mild peripheral erythema on the palmar surface.

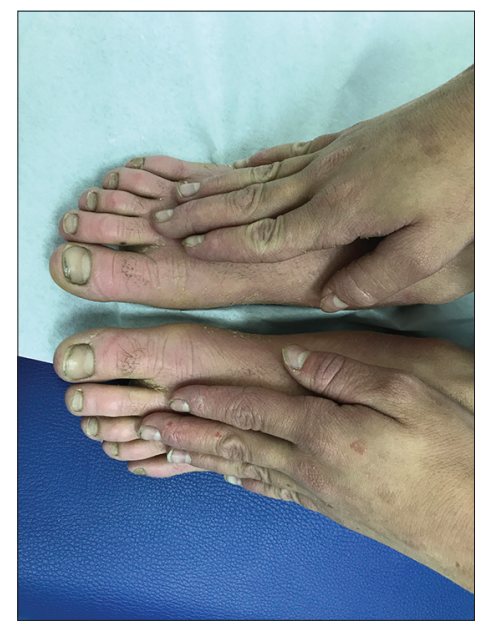

Figure 2: The transgrediens progression of hyperkeratoses to the dorsal surfaces of the hands and feet. renal function test, thyroid function tests, serum iron, total iron binding capacity, urinalysis and stool analysis were within normal range. But total IgE level was high $(5000 \mathrm{IU} / \mathrm{ml})$. ECG and echocardiogram were normal. Biopsy from the trangredient border of keratoderma revealed mild to moderate acanthosis, orthokeratosis, and prominent hypergranulosis without epidermolytic changes. Besides, there was mild perivascular lymphocytic infiltrate in the upper dermis, Interestingly, the presence of less severe hyperkeratosis with the transgredient pattern to the dorsum of the hands and feet, lack of nail involvement and lack of distant keratosis we think that our case is a variant of Mal de Meleda called hereditary PPK of the GamborgNielsen type. Genetic analysis was our next step to detect the affected gene but due its unavailability we decided to postpone and follow the patient every 6 months as a case of Mal de Meleda. Currently, the patient is being treated with strong emollients.

\section{DISCUSSION}

Mal de Meleda is a type of palmoplantar keratoderma (PPK) which has an autosomal recessive (AR) pattern of inheritance with an estimated prevalence of 1 case per 100,000 population [8]. The disease has been reported in many countries including Croatia, Algeria, Chile, China, Germany, India, Indonesia, Italy, Japan, Korea, Laos, Libya, The Netherlands, Pakistan, Saudi Arabia, Scotland, Sweden, Tunisia, Turkey, and the United Arab Emirates [3,9].

Clinically, the patient presents with bilateral diffuse PPK in a stocking and glove pattern with sharp margins and a yellow tone. Usually, symptoms begin soon after birth as palmoplantar erythema and progress to the characteristic thickened, exfoliative hyperkeratosis that has a transgrediens nature [2]. This can be complicated by hyperhidrosis and microbial infection leading to malodorous and painful lesions [1]. Nail anomalies are the most common associated feature in the form of subungual hyperkeratosis, Beau's lines, onycholysis, hyperconvexity, and koilonychias [1,2]. Digital disorders including fifth-finger dysplasia, tapering of the digits, contractures, knuckle pads and pseudoainhum may be present $[2,4,10]$. Other associated features such as psoriasiform lesions on the knees and elbows, perioral erythema, angular cheilitis, arched palate and rarely malignant melanoma within the areas of hyperkeratosis may be seen [3-7]. Histologically, there is hyperkeratosis, parakeratosis, orthokeratosis, 
hypergranulosis and acanthosis without evidence of epidermolysis [11-14]. Mal de Meleda can be diagnosed based on clinical features, family history and genetic analysis. Fischer et al, identified the gene that encodes the secreted LY6/urokinasetype plasminogen activator receptor (uPAR)-related protein-l (SLURP-1) to be the cause [15]. Originally, there were three different gene mutations of SLURP-1 a homozygous single nucleotide deletion, a homozygous point mutation and a homozygous splice site mutation, However, 14 other mutations have been identified in 19 other countries later $[10,16,17]$. Pathophysiologically, when the SLURP-1 protein is non-functional, hyperkeratosis results due to improperly regulated keratinocyte apoptosis and the macrophage and keratinocyte release of tumor necrosis factor (TNF)-a is inhibited producing generalized inflammation $[18,19]$. There are presently over 25 identified hereditary PPK diseases that can be separated into five basic categories: (1) diffuse PPK; (2) diffuse mutilating PPK; (3) focal PPK; (4) ectodermal dysplasia with PPK and (5) syndromic PPK [20]. Specifically, in hereditary PPK of the Gamborg-Nielsen type which was reported in the Swedish population and is referred to as a variant of Mal de Meleda with milder hyperkeratosis, lack of nail involvement, and no distant keratoses. But the transgredient pattern of hyperkeratosis to the dorsum of the hands and feet and knuckle pad formation are variably present [21]. Clinically, our case is similar to the Gamborg-Nielsen type. Though we could not explain the high IgE in the absence of atopy, it could be a new associated finding. Other related conditions include: Naxos syndrome and Carvajal syndrome which are both syndromic PPK diseases with congenital cardiomyopathy, PapillionLefevre syndrome (PLS) is another AR PPK typically with periodontitis and early tooth loss, Nagashimatype PPK is a non-progressive hyperkeratosis usually terminates at puberty, Greither's disease present later in childhood and Olmsted syndrome presents in early childhood with a mutilating-type PPK with a tendency to involve the perioral area $[22,23]$. Treatment include topical corticosteroid, lactic acid, retinoid acid, emollients, keratolytics with topical urea-based ointments and oral 13-cis retinoid acid [9]. Recently, Gruber et al, showed effective treatment with oral acitretin $20 \mathrm{mg}$ /day plus topical antimicrobial and keratolytic therapy [14]. Surgical treatment of hyperkeratosis can be an option with excision and subsequent placement of a full thickness skin graft [5]. Genetic counselling is warranted in these families. The absence of symptoms in biological parents is most likely suggestive of either an AR inheritance pattern or a spontaneous mutation.

Our patient is the first reported case of GamborgNielsen variant of Mal de Meleda in an Egyptian origin patient in the English medical literature. Genetic analysis is being undertaken to confirm the clinical diagnosis.

\section{REFERENCES}

1. Morais e Silva F, Cunha T, Boeno E, Steiner D. Mal de Meleda: a report of two cases of familial occurrence. An Bras Dermatol. 2011;86(4 Suppl 1):S100-3.

2. Marrakchi Z, Marrachi S, Meziou TJ, Turki H, Zahaf A. Mal de Meleda. 16 cases. Tunis Med. 2006;84:423-6.

3. Bchetnia M, Laroussi N, Youssef M, Charfeddine C, Ben Brick AS, Boubaker MS, et al. Particular Mal de Meleda phenotypes in Tunisia and mutations founder effect in the Mediterranean region. Biomed Res Int. 2013;2013:206803.

4. Nath AK, Chaudhuri S, Mohan Thappa DM. Mal de Meleda with lip involvement: a report of two cases. Indian J Dermatol. 2012;57:390-3.

5. Sartore L, Bordignon M, Bassetto F, Voltan A, Tomat V, Alaibac M. Melanoma in skin affected with keratoderma palmoplantaris hereditaria (Mal de Meleda): treatment with excision and grafting. J Am Acad Dermatol. 2009;61:161-3.

6. Mozzillo N, Nunziata CA, Caraco C, Fazioli F, Botti C. Malignant melanoma developing in an area of hereditary palmoplantar keratoderma (Mal de Meleda). J Surg Oncol. 2003;84:229-33.

7. Tjiu JW, Lin PJ, Wu WH, Cheng YP, Chiu HC, Thong HY, et al. SLURP1 mutation-impaired T-cell activation in a family with mal de Meleda. Br J Dermatol. 2011;164:47-53.

8. Fischer J, Bouadjar B, Heilig R, Huber M, Lefevre C, Jobard F. Genetic linkage of Meleda disease to chromosome 8qter. Eur J Hum Genet. 1998;6:542-7.

9. Perez C, Khachemoune A. Mal de Meleda: A Focused Review. Am J Clin Dermatol. 2016;17:63-70.

10. Lestringant GG, Hadi SM, Qayed KI, Blayney BJ. Mal de Meleda: recessive transgressive palmoplantar keratoderma with three unusual facultative features. Dermatology. 1992;184:78-82.

11. Bouadjar B, Benmazouzia S, Prud'homme J, Cure S, Fischer J. Clinical and genetic studies of 3 large, consanguineous, Algerian families with Mal de Meleda. Arch Dermatol. 2000;136:1247-52.

12. Rajashekhar N, Moideen R. Palmoplantar keratoderma-Mal de Meleda syndrome. Int J Dermatol. 1997;36:854-6.

13. Hansen R, Schachner L. Pediatric dermatology. $4^{\text {th }}$ ed. London: Elsevier Health Sciences; 2011.

14. Gruber R, Hennies HC, Romani N, Schmuth M. A novel homozygous missense mutation in SLURP1 causing Mal de Meleda with an atypical phenotype. Arch Dermatol. 2011;147:748-50.

15. Fischer J, Bouadjar B, Heilig R, Huber M, Lefèvre C, Jobard F, et al. Mutations in the gene encoding SLURP-1 in mal de Meleda. Hum Mol Genet. 2001;10:875-80.

16. Eckl KM, Stevens HP, Lestringant GG, Westenberger-Treumann M, Traupe H, Hinz B,et al. Mal de Meleda (MDM) caused by mutations in the gene for SLURP-1 in patients from Germany, Turkey, Palestine, and the United Arab Emirates. Hum Genet. 2003;112:50-6.

17. Charfeddine C, Mokni M, Ben Mousli R, Elkares R, Bouchlaka C, Boubaker $\mathrm{S}$, et al. A novel missense mutation in the gene encoding SLURP-1 in patients with mal de Meleda from northern Tunisia. Br J Dermatol. 2003;149:1108-15.

18. Grando SA, Pittelkow MR, Schallreuter KU. Adrenergic and 
www.odermatol.com

cholinergic control in the biology of epidermis: physiological and clinical significance. J Invest Dermatol. 2006;126:1948-65.

19. Chimienti F, Hogg RC, Plantard L, Lehmann C, Brakch N, Fischer J, et al. Identification of SLURP-1 as an epidermal neuromodulator explains the clinical phenotype of Mal de Meleda. Hum Mol Genet. 2003;12:3017-24.

20. Braun-Falco M. Hereditary palmoplantar keratodermas. J Dtsch Dermatol Ges. 2009;7:971-84 (quiz 984-985).

21. Kastl I, Anton-Lamprecht I, Gamborg Nielsen P. Hereditary palmoplantar keratosis of the Gamborg Nielsen type. Clinical and ultrastructural characteristics of a new type of autosomal recessive palmoplantar keratosis. Arch Dermatol Res. 1990;282:363-70.
22. Athanikar SB, Inamadar AC, Palit A, Sampagavi VV, Deshmukh NS Greither's disease. Indian J Dermatol Venereol Leprol. 2003;69:292-93.

23. Duchatelet $\mathrm{S}$, Hovnanian A. Olmsted syndrome: clinical, molecular and therapeutic aspects. Orphanet J Rare Dis. 2015;17:33.

Copyright by Khalid M. Al-Husain, et al. This is an open-access article distributed under the terms of the Creative Commons Attribution License, which permits unrestricted use, distribution, and reproduction in any medium, provided the original author and source are credited.

Source of Support: Nil, Conflict of Interest: None declared. 